Vol. Vol. 9(14), pp. 329-331, 30 July, 2014

DOI: $10.5897 /$ IJPS2014.4166

ISSN 1992 - 1950

Article Number: 7BA50FA46342

Copyright (C) 2014

Author(s) retain the copyright of this article

http://www.academicjournals.org/IJPS

International Journal of Physical Sciences

\title{
Internuclear forces: Physical and chemical reasons
}

\author{
B. A. Mosienko \\ Siberian Research Institute of Geology, Geophysics and Mineral Resources, Krasnii pr. 67, Novosibirsk, 630104 Russia. \\ Received 21 May, 2014; Accepted 17 July, 2014

\begin{abstract}
The arguments, obtained in favor of the hypothesis on internuclear interaction of atoms (offered by author in the preceding publication), are presented. It is proposed an interpretation of physical phenomenon Bragg peak, which is discovered 100 years ago but is not understood hitherto. An extremely simple and natural explanation of chemical covalent bond (which unlikely may be considered satisfactory at present) is produced.
\end{abstract}

Key words: Internuclear interaction, п0-mesons, Brag peak, chemical bond.

\section{INTRODUCTION}

As is known, the psychological need to live in a comprehensible world is inherent in us primarily. "People are inclined to consider that they know everything that may be known. Such philosophy of life had always been. It is enough to read the works of Newton's contemporaries in order to see that in those times many people believed that with the discovery of classical laws of mechanics, our cognition of inanimate nature was completed" (Kapitsa, 1959). Nevertheless, our notion of surrounding world increases and sometimes essentially changes.

In the preceding works, Mosienko $(2008,2009)$ have propounded and grounded a notion of the new state of matter in atomic-molecular form, which is called second liquid. In order to solve the problem on the character of forces between second liquid molecules, author has proposed a hypothesis on the internuclear interaction of atoms (Mosienko, 2013). Note, that this hypothesis is not an arbitrary author's fantasy; it proceeds from a definite likeness between two levels of matter - nuclear and molecular. Indeed, principal elements of the nuclear level are protons; principal elements of the molecular level are atoms. It is known, that radius of atom is about $10^{5}$ times as much as radius of proton. The effective radius $r$ of central forces is determined by mass $m$ of their carriers:

$r=h / m c$,

where $h$ is Plank constant and $c$ is velocity of light. The carriers of forces between protons in atomic nuclei are $\Pi^{0}$-mesons. By analogy, it is naturally to suppose that the carriers of forces between the neighboring atoms of second liquid molecules are the light and electrically neutral analogs of $\pi^{0}$-mesons that is, some $x^{0}$-mesons (which are given off by protons) with mass, as it follows from Equation (1), about $10^{5}$ times as less as mass of $\pi^{0}-$ meson.

Below it is shown that the proposed hypothesis allows to interpret the physical phenomenon Bragg peak and gives a new explanation of the chemical bonding. 


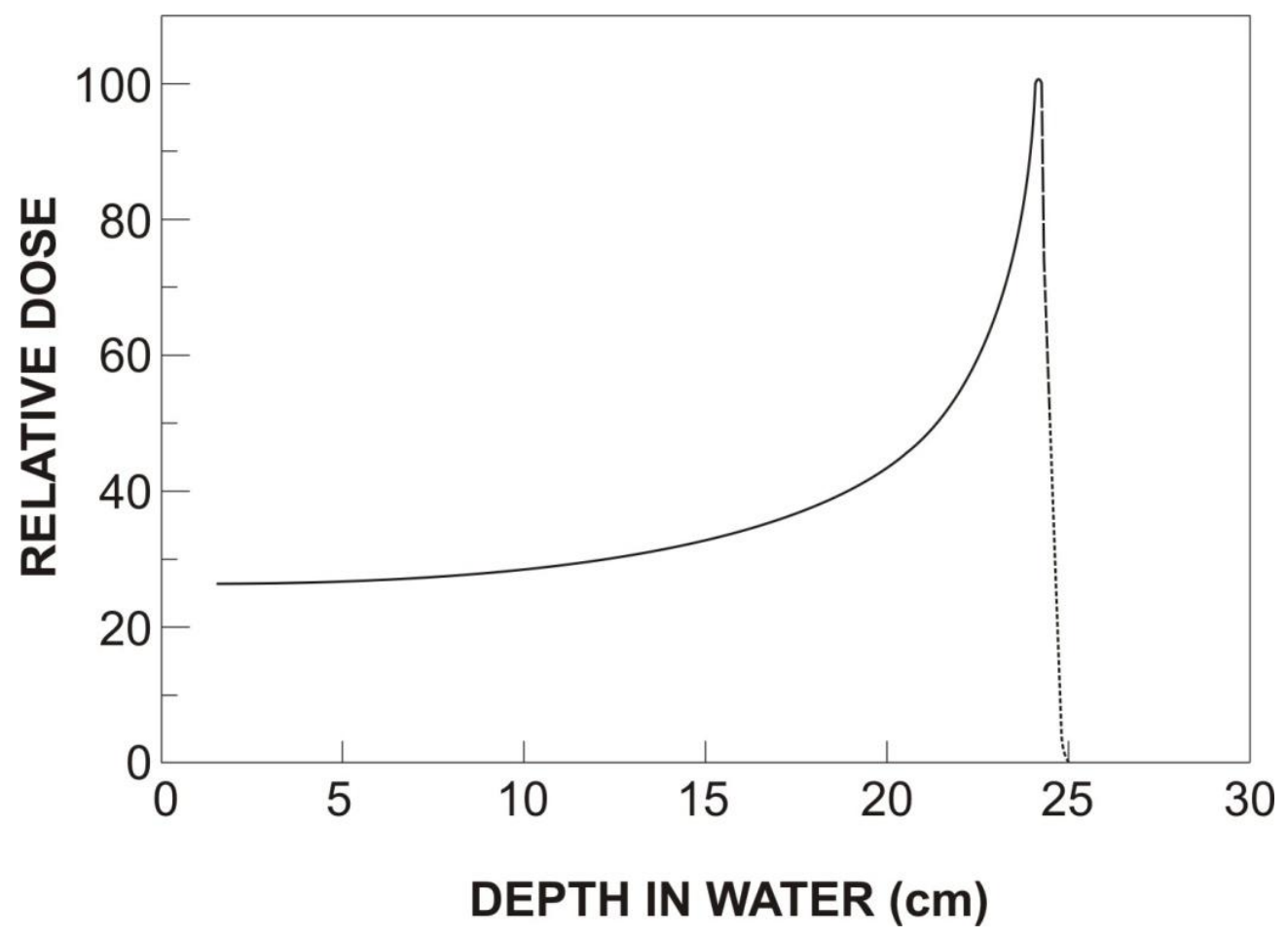

Figure 1. The ionization curve of a proton beam in water (Bragg peak).

\section{THE PHYSICAL PHENOMENON BRAGG PEAK}

Bragg peak (Wagenaar, 1995; Khoroshkov, 2006; Soltani-Nabipour et al., 2009) is a drastically expressed maximum in the end of the curve which represents radiation power dependence of the substance, through which a charged particle (for example, proton) moves, from kinetic energy of this particle (Figure 1). It had gotten its name from well-known English physicist Henry Bragg, who discovered this phenomenon in 1903. Note that Röntgen radiation is the radiation of substance observed in Bragg peak; in the course of some decades, it is employed for medical treatment of malignant formations.

At present, the origin of Bragg peak is explained as follows: the charged particle, in the end of its way, moves slower; so, the interaction cross section of it with substance, which it moves through, increases. Just this increase is observed in the form of peak. It is quite easy to understand that such explanation is unconvinced. Indeed, let us pay attention to the shape of Bragg curve. At first, it slowly rises, and this ascent really could be explained with an increase of the interaction cross section during a decrease of the charged particle velocity. But in the very end, Bragg curve suddenly rises and forms a sharp peak. Such form of the curve testifies that we deal with the resonance curve. But the origin of this resonance remains unknown. Otherwise, the physical essence of this phenomenon is incomprehensible hitherto. Let us endeavor to explain this phenomenon. We know, that the irradiation of atomic nuclei with a bunch of the quickly moving protons (by proton's kinetic energy about $300 \mathrm{MeV}$ ) leads to the appearance of great number $\pi^{0}$-mesons, which, at insignificant share of second, decay into gamma-quanta (Kitaigorodsky, 1973); by this, $\pi^{0}$-meson turns into two gamma-quanta. Just the appearance of great number of gamma-quanta is observed in experiments. Process of gamma-quanta formation has resonance character; the real $\pi^{0}$-meson forms from the virtual one by way of resonance absorption of the half proton kinetic energy (about 150 $\mathrm{MeV}$ ), the second half of energy is taken away by collided particles.

In the case of Bragg peak, an analogous phenomenon is observed: when the proton kinetic energy is reduced approximately to $3000 \mathrm{eV}$, the sudden appearance of great number of Röntgen radiation quanta happens. This radiation also has resonance character. By analogy, we come to conclusion that, during resonance absorption of the half proton kinetic energy, the proposed virtual particle ( $x^{0}$-meson) turns into the real particle and at once decays to the couple of Röntgen photons. We emphasize that the real kinetic energy of protons, corresponding to Bragg peak, is unknown, it could be found just with a special experiment. The above mentioned value (3000 $\mathrm{eV}$ ) is hypothetical (as $300 \mathrm{MeV} / 10^{5}=3000 \mathrm{eV}$ ), but it is quite plausible. As we can see, the hypothesis on the internuclear interaction makes it possible to explain the 
physical essence of Bragg peak phenomenon.

\section{ON THE NATURE OF CHEMICAL BOND}

It is clear enough that our hypothesis can explain not only a bonding between second liquid molecules, but also a chemical bond between atoms in molecules. This problem, in part, is considered in the author's preceding work (Mosienko, 2013); here we intend to continue this theme.

It is known that experimental values of ionic bond are noticeably higher than theoretical ones; this excess is named "nonionic contribution" (Barnard, 1965; ErdeyGruz, 1967). In our opinion, this contribution is mainly indebted to internuclear interaction.

Let us proceed to covalent chemical bond; we consider a bonding of atoms in hydrogen molecule. The quantitative calculation of chemical bonding, for the first time, was fulfilled by German researches W. Heitler and F. London in 1927. Of course, the solution of this problem might be considered as scientific feat. But let us glance at this problem, as far as possible, unprejudicely. In order to initiate bonding between hydrogen atoms, electrons of atoms might be transferred (at any rate, partly) in the internuclear space. It is easy to see that in this case, Coulomb's law would be infringed. In addition, coordination of the electrons movement would be violated. Unnaturalness of such behavior of electrons is obvious. However, we must reconcile ourselves with such interpretation; otherwise, it is impossible to explain the existence of molecules in the frame of existent notions. In the meantime, if one will apply the hypothesis of internuclear interaction of atoms, the problem of covalent bond will be extremely solved in a simple and quite natural manner (Mosienko, 2013).

\section{CONCLUSION}

Thus, the resonance absorption of kinetic energy of protons is observed in the two points: about $300 \mathrm{MeV}$ and about $3000 \mathrm{eV}$. In the first case, we know that transformation of virtual $\pi^{0}$-mesons into the real ones takes place. In the second case, we propose, by analogy, that transformation of virtual $x^{0}$-mesons into the real ones happens. This enables interpretation of Bragg peak phenomenon, which may be considered an argument in favor of the proposed hypothesis. Further convincing argument, from personal perspective is the simplicity and naturalness of covalent bond in the new understanding. It should be emphasized that this hypothesis is propounded from necessity, for explanation of the interaction mechanism between second liquid molecules. The other important results (in particular, essential simplification of chemical bonding mechanism) are received automatically, that is, without supplementary suppositions. Apparently, it means that the proposed idea is something greater than pure hypothesis.

\section{Conflict of Interest}

The authors have not declared any conflict of interest.

\section{REFERENCES}

Barnard AK (1965). Theoretical basis of inorganic chemistry. New York, London, Toronto, Chap. 2,3.

Erdey-Gruz T (1967). Grundlagen der structur der materie. Leipzig, Chap. 5,6.

Kapitsa PL (1959). Speech on Int. Symp. Prague.

Khoroshkov VS (2006). Radiation beam therapy evolution: from x-rays to hadrons. Phys. Atomic Nuclei 69:1724-1742.

Kitaigorodsky Al (1973). Introduction in physics. Moscow, Chap. 31.

Mosienko BA (2008). Sublimation: a two-step phase transition. Z. Phys. Chem. 222:1533-1549.

Mosienko BA (2009). Second liquid - an intermediate phase of sublimation. Ibid. 223:905-919.

Mosienko BA (2013). Possible internuclear interaction of atoms. Int. J. Phys. Sci. 8(37): 1824-1829.

Soltani-Nabipour J, Sardari D, Cata-Danil Gh (2009). Sensitivity of the Bragg peak curves to the average ionization potential of the stopping medium. Rom. J. Phys. 54:321-330.

Wagenaar DJ (1995). Radiation physics principles. Lectures Cycle. Sect. 7. 